\title{
Modelling of shale rock pore structure based on gas adsorption
}

\author{
Arghya Das ${ }^{1, *}$, Sumit Basu², and Ankit Kumar $^{1}$ \\ ${ }^{1}$ Indian Institute of Technology Kanpur, Department of Civil Engineering, Kanpur 208016, India \\ ${ }^{2}$ Indian Institute of Technology Kanpur, Department of Mechanical Engineering, Kanpur 208016, India
}

\begin{abstract}
Shale rock consists of a complex matrix structure due to presence of nano-scale pores. Owing to such complexity determination and/or prediction of the mineralogical, mechanical, and petrophysical properties (e.g., permeability, porosity, pore size distribution, etc.) of shale is a challenging task. A preliminary estimation of these properties is essential before shale gas exploration. In this study, experimental and numerical analyses are conducted to estimate the permeability, porosity, and pore size distribution of a typical shale sample. Gas adsorption experiments were conducted to characterize the pore spaces of the shale via analysing the isotherms. Using conventional theories, such as BET and BJH methods, surface area, pore volume, and pore size distributions were estimated. On the other hand, gross porosity of the shale samples was measured by conducting gas pycnometry experiment. Finally based on the obtained results an equivalent pore network model is constructed which accounts for the pore size distributions and low pore connectivity in the shale matrix. We have simulated gas flow through the network to estimate permeability of the shale. This model considers Knudsen diffusion and the effects of gas slippage on permeability. Further parametric study shows that the apparent permeability primarily depends on the reservoir pressure, pore coordination number and porosity.
\end{abstract}

\section{Introduction}

Due to the increasing energy demand and uprising oil and gas prices in the world, the traditional exploration of the conventional resources has shifted towards unconventional sources. Coal Bed Methane (CBM), Coad Mine Methane (CMM), Shale Gas, and Tight Gas are considered as typical alternative unconventional sources [1]. Prior to gas exploration it is essential to estimate the total gas-in-place within the shale. However, it is a challenging task since, shale is an ultra-thick sedimentary rock having nanoscale pores and a very low permeability in the order of $10^{-25}$ to $10^{-15} \mathrm{~m}^{2}$.

Despite a few advantages of the laboratory based testing, researchers are now advocating the use of micromechanics based multi-scale modelling for shale using pore distribution and clay microstructure. Several geophysical research groups in the United States and China [2-3] pioneered such modelling of shale in recent years. The advantages of these models are manifold in terms of anisotropic stiffness and permeability prediction. Though the basic algorithms for devising such models are well established, the microstructural characterization and numerical implementation are non-trivial and require further insight.

Pore network modelling has achieved a great deal of success in predicting relatively reliable results for shale permeability [4]. Numerous methods are available to construct the pore network models. Nonetheless, imaging techniques (e.g., SEM, TEM, X-ray micro-CT scan, and FIB) for analysing the pore structure and network extraction algorithms are the most common approaches [5-6]. Numerical algorithms, such as medial axis, and maximal ball have proved their importance in pore network extraction after image analysis [7]. Other experimental techniques like gas adsorption and mercury intrusion porosimetry have also been adopted for pore size distribution by many researchers [8]. These two techniques (imaging and gas adsorption) are the most conventional techniques used in pore network modelling for getting pore size distribution.

Here, an attempt has been made to determine the apparent permeability of Indian shale. The main objective of this study is to develop a three-dimensional nano-scale pore network model (PNM) to simulate the gas flow through shale while considering the micro-scale mechanisms like Knudsen diffusion, slip flow, and transition flow. In order to develop such models, pore connectivity and pore-throat size distribution are required. Therefore, a detailed microstructural analysis of the shale is carried out via gas adsorption technique.

\section{Experimental programme}

Pore size, density, and porosity of a shale sample, were analyzed via fluid invasion methods such as $\mathrm{N}_{2}$ adsorption and gas pycnometer tests. Besides porosity, pore volume and surface area were also estimated from these tests.

\footnotetext{
* Corresponding author: arghya@iitk.ac.in
} 


\subsection{Gas pycnometer test}

Gas pycnometer test is carried out to determine the true volume and density of a porous solid specimen. During the test a known volume of non-corrosive gas (e.g. Helium) is allowed to expand within a specimen chamber and the consequent pressures drop is measured, which is used for the determination of specimen volume. Further, the gross porosity of the specimen can also be estimated. Two cylindrical cores were prepared of size $\sim 1 \mathrm{~cm}$ diameter and $\sim 3 \mathrm{~cm}$ height. The test results are given in Table 1. Despite obtaining a closed range density, large variation in the porosity can be noticed here.

Table 1. Gas pycnometer results.

\begin{tabular}{|l|l|l|l|l|}
\hline & Mass $(\mathrm{g})$ & $\begin{array}{c}\text { Volume } \\
(\mathrm{cc})\end{array}$ & $\begin{array}{c}\text { Density } \\
(\mathrm{g} / \mathrm{cc})\end{array}$ & $\begin{array}{c}\text { Porosity } \\
(\%)\end{array}$ \\
\hline Core 1 & 11.78 & 4.23 & 2.73 & 2.24 \\
\hline Core 2 & 11.72 & 4.12 & 2.84 & 6.32 \\
\hline
\end{tabular}

\section{$2.2 \mathrm{~N}_{2}$ adsorption test}

In this analysis, primarily the nano-scale pores are identified by measuring the volume of liquid $\mathrm{N}_{2}$ entrapped within the pores of a powdered specimen/adsorbent under isothermal condition. Four powdered samples (grain size $<25 \mu \mathrm{m}$ and mass around $0.02-0.07 \mathrm{~g}$ ) were prepared from the shale samples. These tests were performed using 'Quanta chrome Autosorb 1C BET Surface Area \& Pore Volume Analyzer' in IIT Kanpur. A typical variation in adsorption (and desorption) volume of gas against relative pressure (in other words Isotherm) for a sample is given in Figure 1.

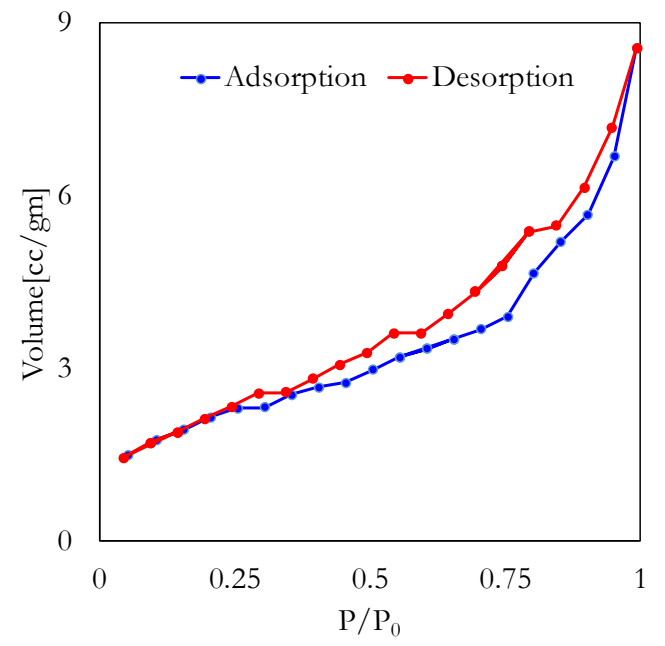

Fig. 1. Typical isotherm of Indian shale.

The isotherms do not show distinct adsorption and desorption paths during the relative pressure variation. Rather a narrow hysteresis can be noticed. Therefore, the isotherms can be classified as Type-II(B) as recommended by Rouquerol et al. [9]. Such a pattern in the isotherms indicates the presence of mesopores ( 2 to 50 $\mathrm{nm})$.

\subsection{Pore size distribution}

Pore size distribution is obtained from the isotherms of the four samples using density functional theory (DFT) for the identification of micropores $(<2 \mathrm{~nm})$ and BarrettJoyner-Halenda (BJH) method for the identification micro- and mesopores $(2 \mathrm{~nm}-50 \mathrm{~nm})$. The pore distribution corresponding to the sample isotherm shown in Figure 1 is given in Figure 2. Pore size distribution indicates that irrespective of the porosity, both the shale specimens contain pores of diameter 3 to $7 \mathrm{~nm}$.

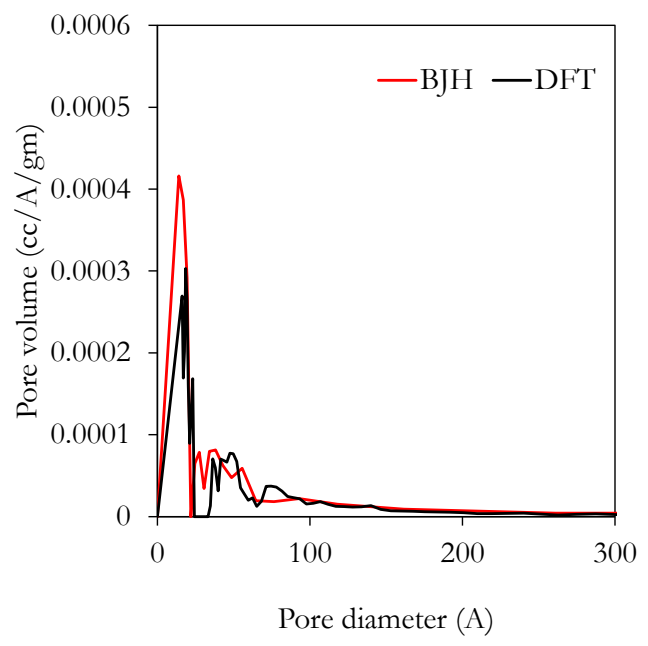

Fig. 2. Pore size distribution obtained from BET experiments.

\section{Pore-network modelling}

In this present study, a three-dimensional pore network model is constructed by using OpenPNM [10], an open source python based numerical modelling tool, which accounts for the various size distribution and low interconnectivity of the pores in shale. We adopt the formulation of Javadpour et al. [11] for such flow simulation. The gas flow module of OpenPNM is upgraded with the effects of gas slippage and Knudsen diffusion.

\subsection{Gas flow equation in the shale matrix}

In the current study, a single fluid phase is considered for the simulations. The fluid flow rate can be formulated by the Darcy flow equation considering gas compressibility; the mass flux equation is given below:

$$
J_{a}=-\frac{\rho r^{2}}{8 \mu} \nabla p
$$

where $\rho$ is the gas density, $\nabla p$ is the pressure gradient; $r$ is the radius of flow tube; and $\mu$ is the dynamic gas viscosity.

However, it is evident from the laboratory scale experiments and physical understandings that flow through the nanoscale channels is significantly governed by the drag along the pore walls, (which is known as the gas slippage) and the collision between the gas molecules and pore internal surface (in other word diffusion) [4]. 
Therefore, the gas migration by considering Knudsen diffusion and gas slippage effects is analysed here.

In shale, the gas diffusion and slip effect both coexist, whereby the flow regime changes from slip to diffusion within the nanoscale pores. In order to identify this transition, Knudsen number is defined as the ratio between the gas molecular mean free path and the characteristics pore size $(r)$.

A correction factor $(F)$ is introduced to consider the gas slippage effect [12];

$$
F=1+\frac{4 \lambda}{r}
$$

where, $\lambda$ is the gas molecular mean free path, which can be expressed by the following relation;

$$
\lambda=\frac{\mu}{p} \sqrt{\frac{\pi R T}{2 M}}
$$

Here $M$ is the gas molar mass; $R$ is the gas constant; $T$ is the absolute temperature; Therefore, the modified gas mass flux after considering the slippage effect is given by

$$
J_{a}=-F \frac{\rho r^{2}}{8 \mu} \nabla p
$$

The diffusion part is incorporated through the Knudsen diffusion model [11] in the following manner,

$$
J_{d}=-\frac{2 r}{3} \frac{M}{R T} \sqrt{\frac{8 R T}{\pi M}} \nabla p
$$

Combining eq. 4 and 5 the total gas mass flux $(J)$ through nano-porous shale become:

$$
J=-\left(F * \frac{\rho r^{2}}{8 \mu}+\frac{2 r}{3} \frac{M}{R T} \sqrt{\frac{8 R T}{\pi M}}\right) \nabla p
$$

The apparent permeability $\left(K_{a p p}\right)$ of the pore network is following,

$$
K_{a p p}=-\left[F \frac{r^{2}}{8}+\frac{2 r}{3} \frac{\mu}{R T} \sqrt{\frac{8 R T}{\pi M}}\right]
$$

On the other hand the intrinsic permeability of network $\left(K_{d}\right)$ is obtained using Darcy's law.

$$
K_{d}=-\frac{r^{2}}{8}
$$

Finally, a correction factor $(f)$, which quantifies the increase in the permeability value after considering Knudsen diffusion and slippage effect in the shale matrix, is defined as the ratio of apparent permeability to intrinsic permeability.

$$
f=\frac{K_{a p p}}{K_{d}}
$$

This formulation is devised from the consideration of Javadpour [11] flow rate equations except the slip part, which is modified as per Klinkenberg [12].

\subsection{Pore network and permeability}

In order to obtain the apparent permeability in shale, a pore network model is constructed using OpenPNM, which mimics the transport properties of any porous material. In the network the pores and throats are considered as spherical and cylindrical, respectively. Transport characteristics of the model are validated against an existing pore network simulation given in Zhang et al. [4] for 7\% porous Marcellus shale. Apparent permeability comparison at different pressures shows a good agreement between the present model and existing data.

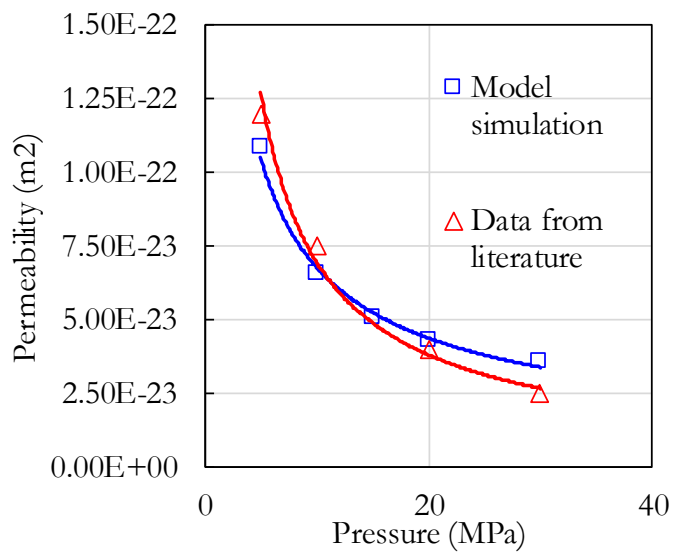

Fig. 3. OpenPNM model validation

In order to determine the permeability characteristics of Indian shale considered in the present study, initially a cubic micro/nano pore network is constructed (shown in Figure 4) with coordination number 20. In this network the pore size distribution follows the distribution obtained from the gas adsorption experiments (BET) as shown in Figure 2. The throat size (diameter) is assumed to have the minimum value of the pore diameter in any pore-throatpore connection. Table 2 collectively shows the parameters and geometry used for the present study. The study assumes Methane as the invading gas. The colour variation within the network in Figure 4 indicates the pressure gradient across the network.

Table 2. Details of the pore network.

\begin{tabular}{|c|c|}
\hline Porosity & $4.2 \%$ \\
\hline Model dimensions & $0.2 \times 0.2 \times 0.2(\mu \mathrm{m})$ \\
\hline Spacing of two consecutive pores & $0.01(\mu \mathrm{m})$ \\
\hline Number of pores in 3 directions & {$[22,22,22]$} \\
\hline Pore size range & $3 \mathrm{~nm}$ to $7 \mathrm{~nm}$ \\
\hline Invading gas phase & Methane \\
\hline Gas temparature & $400 \mathrm{~K}$ \\
\hline Pressure gradient & $1 \mathrm{MPa}$ \\
\hline
\end{tabular}

Since gas adsorption captures only the surface pores irrespective of the pore connectivity, the initial network is constructed with fully connected pores and hence the 
porosity is relatively higher $(10 \%)$ than the shale sample. On the other hand, gas pycnometer only identifies the connected pores and accordingly the porosity is measured. The average connected porosity of the shale is around $4.2 \%$ (Table 1) as measured from the pycnometer test. Therefore, the pore connectivity in the network is trimmed by randomly deleting the throats and the consequently developed isolated pore-throat-pore clusters to achieve the desired porosity.

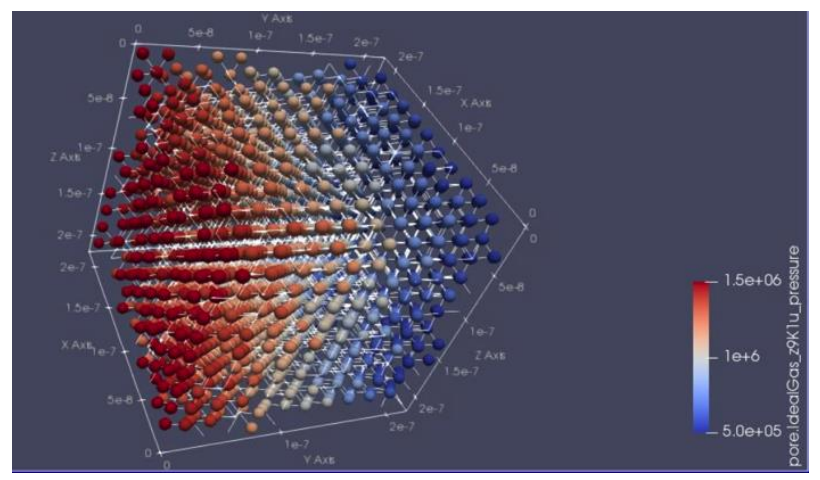

Fig. 4. Equivalent cubic pore network

For flow simulation through the network the modified gas flow model (Eq. 6) is implemented. The discretised form of the mass flux is given by,

$$
\begin{aligned}
& m_{i}^{n}-m_{i}^{n+1}=\sum_{j=1}^{e}\left(F \frac{\pi r^{4}}{8 \mu}\left(\frac{p_{i}{ }^{n}+p_{j}^{n}}{2}\right)+\right. \\
& \left.\frac{2 \pi r^{3}}{3} \sqrt{\frac{8 R T}{\pi M}}\right) \frac{M}{R T} \frac{p_{i}{ }^{n}-p_{j}^{n}}{l_{i j}} \Delta t,
\end{aligned}
$$

Eq. 10 represents the change in gas mass in $i^{\text {th }}$ pore during $n$ to $n+1$ step with step time size $\Delta t$. $i^{\text {th }}$ pore is connected to $\mathrm{e}+1$ numbers of neighbouring pores.

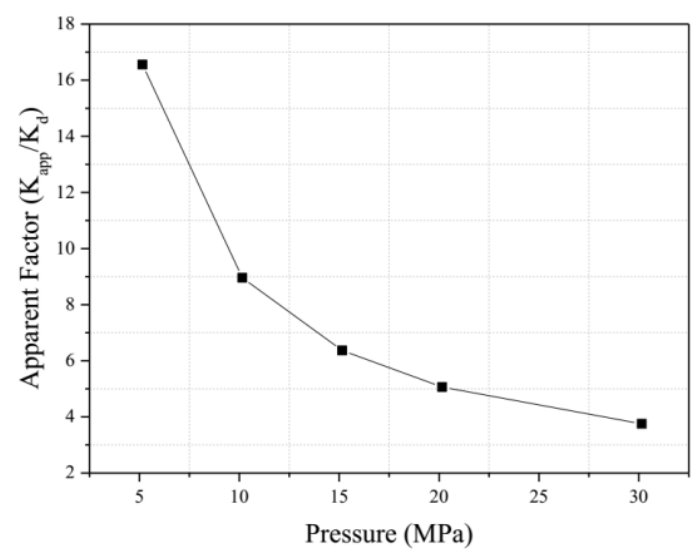

Fig. 5. Permeability variation with pressure

With the developed network three parametric studies are performed, influence of pressure, coordination number namely, connectivity and porosity on permeability. Figure 5 shows the effect of pressure on the apparent permeability factor $(f)$. In low pressure zone $(<10 \mathrm{MPa})$ the apparent permeability is relatively high, 16 times the Darcy permeability, because of higher value of molecular mean free path of the gas molecules, which results in Knudsen diffusion and gas slippage along the pore wall. As the pressure increases the apparent permeability converges to Darcy's permeability since at the low-pressure regime the viscous characteristics dominate. The simulation indicates that the apparent factor starts approaching unity at higher-pressure values $(>30 \mathrm{MPa})$.

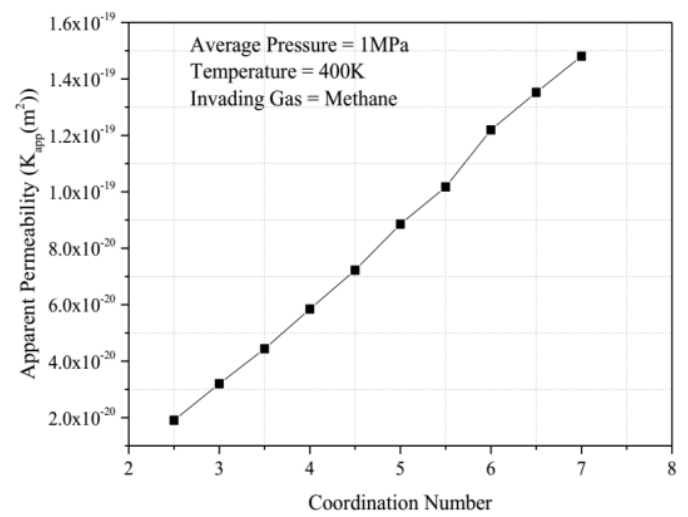

Fig. 6. Influence of coordination number on permeability

Effects of coordination number and porosity on the shale permeability are estimated under $1 \mathrm{MPa}$ average reservoir pressure. Figure 6 , shows the dependence of coordination number (connectivity) on the apparent permeability. The gas flow simulation is conducted for coordination number ranging from 2.5 to 7 with $1 \mathrm{MPa}$ average pore pressure. It can be noted from the plot that the apparent permeability is linearly increasing with the coordination number. Higher coordination number represents more intrinsic connectivity between the pores, which provides a well-connected path for the gas through the shale. Notice that, the permeability of the shale sample is in the range of $10^{-19}$ to $10^{-20} \mathrm{~m}^{2}$. On the other hand, porosity shows a nonlinear correlation with permeability (see Figure 7). Below $10 \%$ porosity permeability significantly reduces, around 2 orders of magnitude.

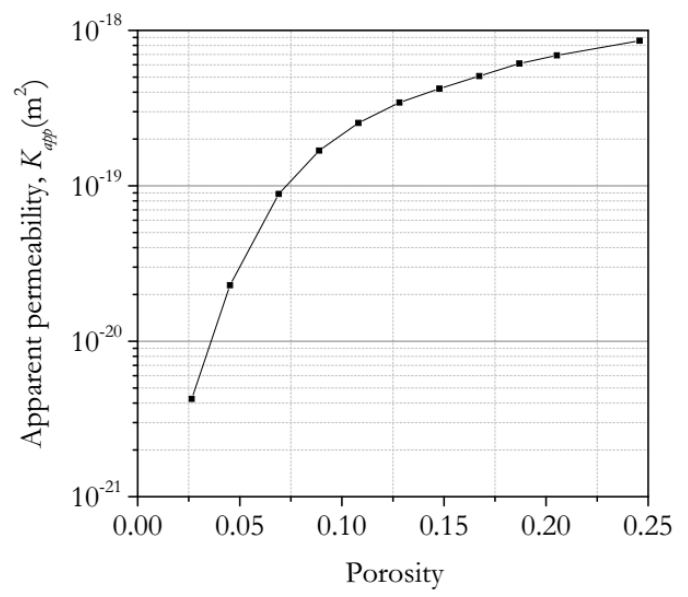

Fig. 7. Influence of porosity on permeability at $1 \mathrm{MPa}$ pressure

\section{Conclusions}

The present study explores pore structure and permeability in Indian shale rock obtained from an anonymous reservoir. Gas adsorption based experimental analysis was performed to determine the pore size distribution, while the porosity was measured via pycnometer. Finally, the results were used to develop a 
pore network model for permeability estimation, while accounting Knudsen diffusion and gas slippage.

Based on the type of isotherms obtained from gas adsorption analysis, the shale sample is considered to have majorly mesopores. The mean pore size of the shale samples is estimated by two different methods (BET and $\mathrm{BJH}$ ), which shows a range between $3 \mathrm{~nm}$ and $7 \mathrm{~nm}$. Gas adsorption data indicates that the average in porosity around $4 \%$.

The results from the model study indicate that the apparent permeability of the studied shale rock is of the order of $10^{-20} \mathrm{~m}^{2}$. However, the parametric study shows permeability can be further reduced with increasing reservoir pressure and decreasing porosity. Further, a linear correlation between permeability and network coordination number is obtained.

The authors wish to thank Oil and Natural Gas Corporation Limited (ONGC), India for providing shale specimen for this study.

\section{References}

1. M. Ahmad, Australian School of Petroleum, (Master Thesis, 2014)

2. J. Shaw, M. Reynolds, and L. Burke, Proc. Can. Int. Pet. Conf. 1, (2006)

3. M. M. Elgmati, H. Zhang, B. Bai, R. E. Flori, and Q. Qu, North Am. Unconv. Gas Conf. Exhib. (2011)

4. P. Zhang, L. Hu, J. N. Meegoda, and S. Gao, Sci. Rep. (Nat. Publ. Gr.) 5, (2015)

5. D. Wildenschild, C. Vaz, M., Rivers, D. Rikard, and

B. Christensen, J. Hydrol. 267 (2002)

6. R.A. Ketcham, and W.D. Carlson, Comput. Geosci. 27 (2001)

7. A.S. Al-Kharusi, and M.J. Blunt, J. Pet. Sci. Eng. 56 (2007)

8. U. Kuila and M. Prasad, Geophys. Prospect. 61, (2013)

9. J. Rouquerol, F. Rouquerol, P. Llewellyn, G. Maurin, and K. Sing, Adsorption by Powders and Porous Solids, 2nd ed. (2013).

10. J. Gostick, M. Aghighi, J. Hinebaugh, T. Tranter, M. A. Hoeh, H. Day, B. Spellacy, M. H. Sharqawy, A. Bazylak, A. Burns, W. Lehnert, and A. Putz, Comput. Sci. Eng. 18, (2016).

11. F. Javadpour, J. Can. Pet. Technol. 48, (2009).

12. L.J. Klinkenberg, Drilling and Production practice, (NY), 1941. 